02

\title{
Особенности спектров гигантского комбинационного рассеяния 4,4'-бипиридина в режиме детектирования единичных молекул
}

\author{
(C) А.М. Полуботко ${ }^{1}$, Е.В. Соловьева ${ }^{2}$ \\ ${ }^{1}$ Физико-технический институт им. А.Ф. Иофрфе, \\ 194021 Санкт-Петербург, Россия \\ ${ }^{2}$ Санкт-Петербургский государственный университет, Институт химии, \\ 198504 Петергоф, Санкт-Петербург, Россия \\ e-mail: alex.marina@mail.ioffe.ru; solovyeva.elena.v@gmail.com
}

Поступила в редакцию 11.05.2017 г.

Кратко описаны основные положения теории спектров гигантского комбинационного рассеяния применительно к режиму детектирования единичных молекул, когда усиление рассеяния может достигать значений $\sim 10^{14}-10^{15}$. Анализ спектров молекул 4,4'-бипиридина, адсорбированных на димерной решетке из острых наночастиц, указывает, что наблюдающееся усиление обусловлено исключительно сильным квадрупольным взаимодействием, проявляющимся в присутствии только линий, обусловленных колебаниями с единичными неприводимыми представлениями групп симметрии $D_{2}$ или $D_{2 h}$, одной из которых описывается молекула. Исследование спектров, полученных методом спектроскопии комбинационного рассеяния, усиленного острием, показывает, что сильное квадрупольное взаимодействие по-прежнему играет определяющую роль, однако сильное дипольное взаимодействие все же проявляется в спектрах в виде слабых запрещенных линий. Этот результат, по-видимому, связан с принципиально другой геометрией эксперимента.

DOI: $10.21883 /$ OS.2018.01.45355.113-17

\section{1. Введение}

Исследование оптических явлений на молекулах, адсорбированных на поверхности металлов, представляет огромный интерес как с фундаментальной, так и с прикладной точек зрения. Особую ценность представляет исследование спектров гигантского комбинационного рассеяния света (ГКР) в режиме, когда становится возможным детектирование малого количества или даже одной молекулы $[1,2]$. На сегодняшний день многие исследователи считают, что причинами ГКР являются электродинамический механизм, обусловливающий величину усиления $\sim 10^{4}$, и химический механизм, обусловливающий дополнительное усиление $\sim 10^{2}$ в первом слое адсорбированных молекул. При этом электродинамический механизм обычно связывают с возбуждением так называемых поверхностных плазмонов. В [3,4] нами было показано, что представление о „химическом“ механизме ошибочно, а дополнительное усиление $\sim 10^{2}$ имеет чисто электродинамическую природу и связано с очень большим различием в усилении электрического поля и его производных в первом и втором слоях адсорбированных молекул в областях поверхности металлов с очень большой кривизной. Это так называемый эффект громоотвода (lightning rod effect). В настоящее время он является основой для нового спектроскопического метода, спектроскопии усиленной острием (tip enhanced Raman spectroscopy, TERS). Кроме того, нами было показано (например, в [3]), что огромное усиление в ГКР связано не только с усилением электрического поля, но и с существованием так называемого сильного квадрупольного взаимодействия, обусловленного огром- ным увеличением одноименных производных компонент электрического поля $\frac{\partial E_{\alpha}}{\partial x_{\alpha}}$, а также особой ролью квадрупольных моментов с одинаковыми индексами $Q_{e x x}, Q_{e y y}$ и $Q_{e z z}$. При этом оно может быть сильнее дипольного и при определенных условиях играет определяющую роль в процессах усиления. В работе [5] с помощью грубой оценки коэффициентов усиления было показано, что усиление в ГКР в режиме детектирования единичных молекул обусловлено исключительно сильным квадрупольным взаимодействием, в то время как усиление электрического поля и связанное с ним сильное дипольное взаимодействие не играет большой роли. В настоящей работе мы покажем, что результат, полученный в [5], подтверждается анализом спектров симметричных молекул, а именно особенностями спектров ГКР 4,4'-бипиридина, полученными в соответствующих условиях. Однако в случае применения для детектирования единичных молекул техники TERS дипольное взаимодействие оказывается сильным по сравнению с квадрупольным и все же проявляется в спектрах.

\section{2. Основные положения дипольно-квадрупольной теории ГКР}

Дипольно-квадрупольная теория поверхностно усиленных оптических процессов описана достаточно подробно в наших работах. Так, теория ГКР опубликована в монографии [3], теория поверхностно усиленного Гипер комбинационного рассеяния (усиленное Гипер КР) достаточно подробно изложена в [6], а теория 
поверхностно усиленного инфракрасного поглощения (УИП) в [7]. Там же читатель может найти ссылки для более детального ознакомления с различными аспектами этих теорий. Поэтому здесь изложим только основные моменты, которые важны для данной работы.

Как известно, гамильтониан взаимодействия света с молекулами (с электронами молекулы) в общем виде имеет вид

$$
\widehat{\mathbf{H}}_{e-r}=-\sum_{i} \frac{i e \hbar}{m c} \mathbf{A}_{i} \nabla_{i}
$$

Здесь суммирование по $i$ есть суммирование по всем электронам, $\mathbf{A}_{i}$ - вектор-потенциал электромагнитного поля в точке расположения $i$-го электрона. Остальные обозначения общепринятые. Учитывая малые размеры молекулы и разлагая векторный потенциал в ряд, гамильтониан (1) может быть преобразован и записан в виде

$$
\widehat{\mathbf{H}}_{e-r}=|\mathbf{E}| \frac{\left(\mathbf{e}^{*} \mathbf{f}_{e}^{*}\right) e^{i \omega t}+\left(\mathbf{e f}_{e}\right) e^{-i \omega t}}{2},
$$

где $\mathbf{E}-$ напряженность электрического поля, $\mathbf{e}-$ вектор поляризации, $\omega-$ частота электромагнитного поля, $\mathbf{f}_{e}$ - обобщенный вектор взаимодействия света с электронами, компоненты которого имеют вид

$$
f_{e \alpha}=d_{e \alpha}+\frac{1}{2 E_{\alpha}} \sum_{\beta} \frac{\partial E_{\alpha}}{\partial x_{\beta}} Q_{e \alpha \beta} .
$$

Здесь $d_{e \alpha}$ и $Q_{e \alpha \beta}$ есть $\alpha$ - и $\alpha \beta$-компоненты соответственно дипольного момента и тензора квадрупольных моментов электронов.

Как известно, в обычной спектроскопии учитывается только дипольное взаимодействие, так как члены в (3), связанные с квадрупольным взаимодействием, очень малы. Однако в поверхностно усиленной спектроскопии, поскольку взаимодействие происходит с поверхностным полем, а не с полем в свободном пространстве, характерным размером изменения электрического поля вблизи поверхности является величина $l$, имеющая смысл характерного размера шероховатостей. При этом роль квадрупольного взаимодействия резко возрастает из-за сильного увеличения производных $\frac{\partial E_{\alpha}}{\partial x_{\alpha}}$, а также особой роли квадрупольных моментов $Q_{e \alpha \alpha}$, имеющих постоянный знак. Это происходит из-за того, что матричные элементы этих моментов, которые определяют такие физические величины, как сечение рассеяния или поглощения, должны быть в среднем значительно больше, чем матричные элементы моментов $Q_{e \alpha \beta}(\alpha \neq \beta)$, поскольку подынтегральные выражения в таких элементах являются „менее осциллирующими“. Указанный эффект есть проявление некоторой „квантовой интерференции“ в том смысле, что связан с необходимостью квантовомеханического усреднения и вычисления матричных элементов операторов квадрупольных моментов, степень осцилляции которых в пространстве зависит от знака и становится большей для операторов с меняющимся знаком, что должно приводить к сильному уменьшению их значений. В дальнейшем моменты $Q_{e \alpha \alpha}$ будем называть основными квадрупольными моментами $Q_{\text {main }}$. То же можно сказать относительно дипольного взаимодействия. Поскольку дипольные моменты являются знакопеременными величинами, то роль квадрупольного взаимодействия с моментами $Q_{e \alpha \alpha}$ в сравнении с ролью дипольного взаимодействия с моментами $d_{\alpha}$ возрастает сильнее, нежели считалось ранее.

Как известно (например, [3]), вблизи таких особенностей шероховатой поверхности металлов, как области с большой положительной кривизной, происходит очень большое усиление компоненты $E_{z}$, перпендикулярной поверхности и ее производной $\frac{\partial E_{z}}{\partial z}$. Так, например, вблизи такой модели шероховатости, как острие, радиальная компонента напряженности электрического поля $E_{r}$ имеет вид

$$
E_{r} \sim\left|\mathrm{E}_{\mathrm{inc}}\right| C_{0}\left(\frac{l_{1}}{r}\right)^{\beta},
$$

и $E_{r} \rightarrow \infty$ при $r \rightarrow 0 \quad(r-$ расстояние до вершины острия). Здесь $\left|\mathrm{E}_{\mathrm{inc}}\right|-$ амплитуда напряженности падающего электрического поля, $C_{0} \sim 1-$ некоторый численный коэффициент, связанный с геометрией эксперимента, $l_{1}-$ характерный размер острия, $0<\beta<1$ и зависит от угла при вершине и комплексной диэлектрической проницаемости острия.

Мерой роли квадрупольного взаимодействия по отношению к дипольному может быть взята величина

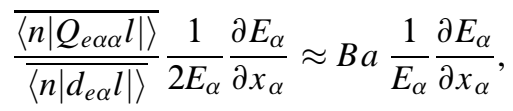

где

$$
B a=\frac{\overline{\left\langle n\left|Q_{e \alpha \alpha} l\right|\right\rangle}}{\overline{\left\langle n\left|d_{e \alpha} l\right|\right\rangle}}
$$

есть отношение некоторых средних значений матричных элементов квадрупольных и дипольных моментов, $a-$ размер молекулы, $B \gg 1$ есть некоторый численный коэффициент, связанный с тем, что квадрупольные моменты $Q_{e \alpha \alpha}$ имеют постоянный, а дипольные моменты $d_{e \alpha}-$ переменный знаки.

Грубая оценка для таких молекул, как, например, бензол, пиридин или пиразин, дает величину $B \sim 2 \cdot 10^{2}$ [3], a для молекулы кристаллического фиолетового (CV), $B \sim\left(1.15 \cdot 10^{2}-2.7 \cdot 10^{2}\right)$ [5]. Отметим, что в соответствии с (6) это достаточно грубая оценка, зависящая от $\alpha$. Однако нам достаточно того факта, что она составляет большую величину $\sim 10^{2}$. Учитывая, что ГКР является процессом второго порядка, то есть состоит из процессов поглощения и спонтанного испускания света, усиление в ГКР за счет чисто дипольного взаимодействия составляет

$$
G_{d} \sim C_{0}^{4}\left(\frac{l_{1}}{r}\right)^{4 \beta}
$$


а за счет чисто квадрупольного взаимодействия

$$
G_{Q} \sim \beta^{4} C_{0}^{4}\left(\frac{B}{2}\right)^{4}\left(\frac{l_{1}}{r}\right)^{4 \beta}\left(\frac{a}{r}\right)^{4},
$$

при этом их отношение

$$
\frac{G_{Q}}{G_{d}} \sim \beta^{4}\left(\frac{B a}{2 r}\right)^{4} .
$$

Из (7), (8) видно, что усиление как за счет чисто дипольного, так и чисто квадрупольного взаимодействия пропорционально степени $4 \beta$ размеров острия $l_{1}$, четвертой степени размера молекулы $a$, и тем больше, чем больше эти величины. Отметим, что этот результат есть следствие электростатического приближения и хорошо согласуется с экспериментальными наблюдениями единичных молекул, которые наблюдались на больших коллоидных частицах с характерным размером $\sim 100 \mathrm{~nm}$ и больших молекулах, таких как родамин желтый (R6G) или кристаллический фиолетовый. Усиление дипольного и в особенности квадрупольного взаимодействия очень сильно возрастает при приближении к вершине. При этом при достаточно малых $r$ таких, что

$$
r<\beta \frac{B a}{2},
$$

квадрупольное взаимодействие становится больше дипольного. Исходя из (7)-(10), можно показать, что для острия с размером $l_{1} \sim 100 \mathrm{~nm}, B \sim 2 \cdot 10^{2}, a \sim 0.5 \mathrm{~nm}$, $C_{0} \sim 1, \beta \approx 1$ усиление за счет квадрупольного взаимодействия $\sim 10^{14}$ достигается при значениях $r \sim 1.26 \mathrm{~nm}$. Это является вполне разумной величиной, при которой как поле, так и его производные, по-видимому, имеют адекватные значения, достижимые в эксперименте. При этом усиление за счет чисто дипольного взаимодействия меньше, чем за счет квадрупольного взаимодействия в $2.4 \cdot 10^{6}$ раз. Видно, что учитывая очень сильную зависимость квадрупольного взаимодействия от расстояния $\sim 1 / r^{4+4 \beta}$, даже некоторое изменение параметров $C_{0}$ и $\beta$ можно скомпенсировать небольшим изменением $r$. При этом чтобы получить значение усиления за счет квадрупольного взаимодействия $\sim 10^{14}$, значение $r$ останется в разумных пределах, а усиление за счет дипольного взаимодействия фактически можно не учитывать.

Как было показано, например, в [3], сечение рассеяния для симметричных молекул в ГКР определяется суммой различных вкладов в рассеяние через различные комбинации дипольных и квадрупольных моментов $T_{(s, p), f_{1}-f_{2}}$ и имеет вид

$$
d \sigma_{\mathrm{SERS}_{s}} \sim \sum_{p} \sum_{f_{1}, f_{2}} T_{(s, p), f_{1}-f_{2}} .
$$

Там же в [3] читатель может найти явное выражение для сечения рассеяния, которое очень громоздко и поэтому здесь не приводится.

В формуле (11) $s, p$ есть квантовые числа соответствующей вырожденной колебательной моды: $s$ -

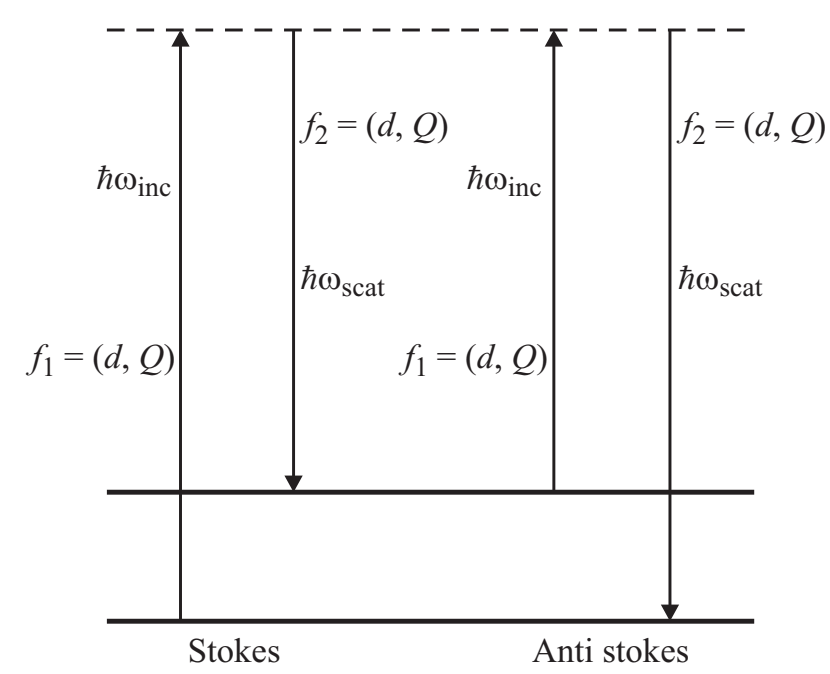

Рис. 1. Диаграмма рассеяния в ГКР. Рассеяние может происходить через различные комбинации дипольных и квадрупольных моментов $d$ и $Q$.

квантовое число, нумерующее группу вырожденных состояний, $p$ нумерует состояния внутри группы. Вклады $T_{(s, p), f_{1}-f_{2}}$ определяют отдельные процессы рассеяния, происходящего через комбинацию дипольных и квадрупольных моментов $f_{1}$ и $f_{2}$ (рис. 1 ). В соответствии с [3] эти вклады подчиняются правилам отбора

$$
\Gamma_{(s, p)} \in \Gamma_{f_{1}} \times \Gamma_{f_{2}}
$$

где $Г$ означает неприводимое представление группы симметрии молекулы, по которому преобразуется колебательная мода $(s, p)$ и дипольные и квадрупольные моменты $f_{1}$ и $f_{2}$. В данной работе, поскольку в дальнейшем будем рассматривать молекулу 4,4'-бипиридина, для которой в группах симметрии молекулы $D_{2}$ или $D_{2 h}$ неприводимые представления одномерны, вырождение отсутствует. Соответственно квадрупольные моменты $Q_{e x x}, Q_{e y y}$ и $Q_{e z z}$ преобразуются по единичному неприводимому представлению как в группе $D_{2}$, так и в группе $D_{2 h}$.

Детектирование единичных молекул относится к случаю очень сильного усиления, когда квадрупольное взаимодействие с основными моментами должно быть значительно больше даже усиленного дипольного взаимодействия. Обозначим вклады в сечение $T_{(s, p), f_{1}-f_{2}}$ просто как $\left(f_{1}-f_{2}\right)$. В соответствии с представлениями, изложенными выше, наиболее усиленными вкладами будут те, которые обусловлены рассеянием на двух основных моментах, то есть $\left(Q_{\text {main }}-Q_{\text {main }}\right)$. Также усиленными, но в значительно меньшей степени, должны быть вклады типа $\left(Q_{\text {main }}-d_{\alpha}\right)$, обусловленные рассеянием через один основной квадрупольный момент и один дипольный момент, и $\left(d_{\alpha}-d_{\beta}\right)$, в частности $\left(d_{\alpha}-d_{\alpha}\right)$. В случае детектирования малого количества или единичных молекул мы должны рассматривать все дипольные моменты, поскольку, как правило, в этих экспериментах моле- 
кулы находятся не в адсорбированном, а в свободном состоянии в растворе с очень малой концентрацией $[1,2]$. В этом случае ориентация молекул может быть произвольной, и усиленное электрическое поле может иметь проекции на все направления системы координат, связанной с молекулой, и все члены гамильтониана дипольного взаимодействия, а также вклады типа $\left(Q_{\text {main }}-d_{\alpha}\right)$ и $\left(d_{\alpha}-d_{\beta}\right)$ будут усилены. Однако поскольку в режиме детектирования малого количества или единичных молекул сильное дипольное взаимодействие значительно меньше, чем сильное квадрупольное, то и эти вклады будут значительно меньше. В соответствии с правилами отбора $(12)$, поскольку $Q_{\text {main }}$ преобразуются по единичному неприводимому представлению, наиболее усиленные вклады будут определять наиболее усиленные линии, относящиеся также к единичному неприводимому представлению. Вклады типа $\left(Q_{\text {main }}-d_{\alpha}\right)$ относятся к неприводимым представлениям, описывающим трансформационные свойства компонент дипольного момента $d_{\alpha}$. Отметим, что вклады типа $\left(d_{\alpha}-d_{\beta}\right)$ и, в частности, вклады типа $\left(d_{\alpha}-d_{\alpha}\right)$, которые также должны определять интенсивности линий, обусловленных колебанием с единичным неприводимым представлением, должны быть малы и в дальнейшем можно их не учитывать. Обычно в ГКР в молекулах с достаточно высокой симметрией вклады типа $\left(Q_{\text {main }}-d_{\alpha}\right)$ определяют появление запрещенных линий, которые активны в инфракрасном поглощении. В нашем случае, поскольку эти вклады значительно меньше, чем вклады типа $\left(Q_{\text {main }}-Q_{\text {main }}\right)$, эти линии должны практически отсутствовать. Отметим однако, что вышеуказанные представления применимы при рассмотрении детектирования единичных молекул на достаточно удаленных друг от друга наношероховатостях, имеющих заострения, когда такие шероховатости можно моделировать острием. В работе [8] детектирование единичных молекул 4,4'-бипиридина проводилось с помощью техники TERS. В связи с этим, по-видимому, конечные результаты измерения спектров и соответственно наши выводы несколько отличаются.

\section{3. Анализ спектров ГКР 4,4'-бипиридина в режиме детектирования малого количества или единичных молекул}

Совместный анализ спектров ГКР, усиленного Гипер КР, КР и инфракрасного поглощения молекулы 4,4'-бипиридина был проведен в работе [9]. Как указано выше, в настоящее время существует две точки зрения на структуру этой молекулы. Согласно [10] она состоит из двух бензольных колец, связанных между собой связью C-C через атомы углерода, в которых атомы углерода в 4,4'-положении замещены на атомы азота. При этом бензольные кольца повернуты относительно друг друга на угол $38.7^{\circ}$ (рис. 2) и группа симметрии молекулы есть $D_{2}$. Согласно другому мнению эти кольца

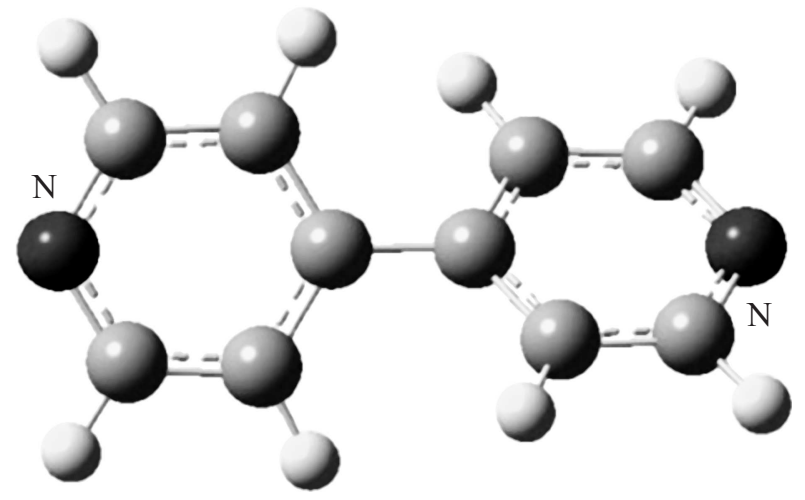

Рис. 2. Возможная геометрия молекулы 4,4'-бипиридина. Кольца повернуты по отношению друг к другу на угол $38.7^{\circ}$, группа симметрии молекулы $D_{2}$.

лежат в одной плоскости и группа симметрии есть $D_{2 h}$. Отметим сразу же, что в дальнейшем будем указывать значения волновых чисел, наблюдающихся в экспериментальных спектрах. При этом в разных экспериментах положение линий может меняться в пределах до $\sim 10-20 \mathrm{~cm}^{-1}$. Поэтому далее, при соотнесении некоторых линий, отличающихся в указанных пределах, мы указываем одно и то же неприводимое представление.

В [9] было показано, что как в спектре ГКР, так и в спектре усиленного Гипер КР большинство линий может быть отнесено как к колебаниям с единичными неприводимыми представлениями $A_{1}$ или $A_{g}$, так и с представлениями $B_{1}$ или $B_{1 u}$. Это связано с особенностями геометрии молекулы, когда два ее кольца могут колебаться либо в фазе, либо в противофазе. Это линии с примерными значениями волновых чисел 396, 1018, 1064-1080, 1208, $1609 \mathrm{~cm}^{-1}$. При этом частоты этих колебаний очень близки и эти состояния являются „Почти вырожденными“ симметричным и антисимметричным состояниями, а поэтому могут быть почти неразличимы в эксперименте. Однако сразу отметим, что в случае детектирования малого числа или единичных молекул вклады, связанные с колебаниями с симметрией $B_{1}$ или $B_{1 u}$, малы, и поэтому в этом режиме упомянутые линии фактически определяются только вкладами, связанными с колебаниями, преобразующимися по единичному неприводимому представлению. Линии с волновыми числами 736, 1300 и $1516 \mathrm{~cm}^{-1}$ относятся только к колебаниям с единичными неприводимыми представлениями $A_{1}$ или $A_{g}$, в то время как линии с волновыми числами 864, 1038 и $1487 \mathrm{~cm}^{-1}$ относятся только к неприводимым представлениям $B_{1}$ или $B_{1 u}$, описывающим трансформационные свойства компоненты дипольного момента $d_{z}$ (здесь ось $z$ проведена так, что соединяет противоположные атомы азота). Спектр 4,4'-бипиридина, адсорбированного из раствора на двумерной решетке из серебряных наночастиц (рис. 3), когда авторы считали, что имеют дело с малым числом, либо даже с единичными молекулами, исследовался 


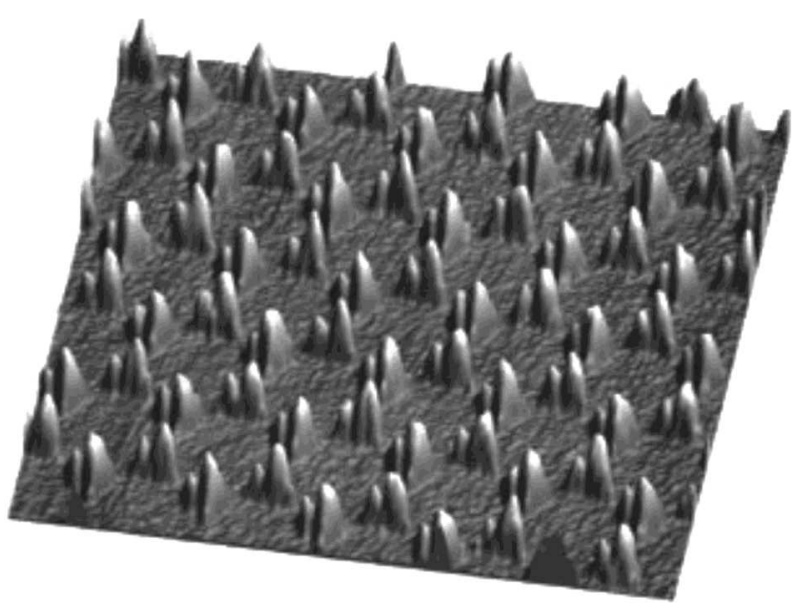

Рис. 3. Вид типичной двумерной нанорешетки из серебра [11].

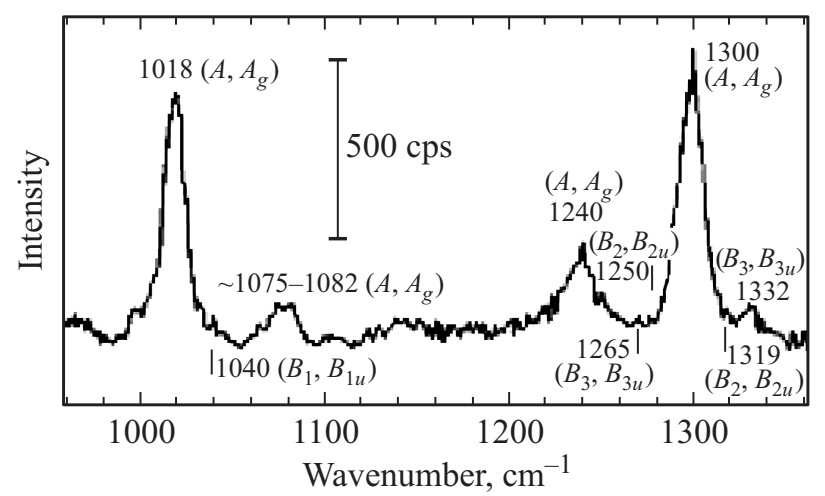

Рис. 4. Спектр ГКР малого числа молекул 4,4'-бипиридина, адсорбированных на двумерной решетке из серебра [11].

в [11] (рис. 4). Сразу отметим, что с нашей точки зрения использование димерных шероховатостей не является принципиальным. Принципиальным является именно наличие областей заострений, где поведение поля будет „близко к сингулярному“.

Необходимо отметить, что в [11] исследовался достаточно узкий интервал волновых чисел $960-1360 \mathrm{~cm}^{-1}$. Поэтому в него попали только следующие линии, относящиеся к колебаниям, преобразующимся как компонента дипольного момента $d_{\alpha}$ : линия $1040 \mathrm{~cm}^{-1}$, относящаяся к неприводимым представлениям $B_{1}$ или $B_{1 u}$, линии 1250 и $1319 \mathrm{~cm}^{-1}$, относящиеся к неприводимым представлениям $B_{2}$ или $B_{2 u}$, и линии 1265 и $1332 \mathrm{~cm}^{-1}$, относящиеся к неприводимым представлениям $B_{3}$ или $B_{3 u}$. Из рис. 4 видно, что эти линии, относящиеся к неприводимым представлениям, описывающим трансформационные свойства дипольных моментов, очень слабы. Этот результат говорит о том, что дипольное взаимодействие все же проявляющееся в этой системе, еще достаточно для наблюдения этих линий, поскольку их интенсивность определяется усреднением по достаточно большому объему, где сильное дипольное взаимодействие может сравниваться с сильным квадрупольным взаимо- действием. Однако их малая интенсивность уже говорит, что в определенных областях сильное квадрупольное взаимодействие значительно больше дипольного. Этот результат хорошо соответствует нашим представлениям. При этом в спектре присутствуют достаточно сильные линии с волновыми числами 1018, 1080 (1064), 1218-1240, 1298-1300 $\mathrm{cm}^{-1}$, в которые есть вклады, относящиеся к колебаниям с единичными неприводимыми представлениями $A_{1}$ или $A_{g}$. Такой же результат был получен в работе [12] (рис. 5), где авторы наблюдали линии 1018, 1078, 1240 и $1300 \mathrm{~cm}^{-1}$ в спектрах ГКР в режиме детектирования единичных молекул на решетке хорошо изолированных наношероховатостей. При этом линии, относящиеся к неприводимым представлениям $B_{1}, B_{2}, B_{3}$ или соответственно $B_{1 u}, B_{2 u}, B_{3 u}$, как и в первом случае отсутствуют. Эти результаты подтверждают тот факт, что усиление в ГКР в режиме детектирования единичных молекул обусловлено почти исключительно сильным квадрупольным взаимодействием. Однако надо отметить, что этот вывод справедлив для наношероховатостей, имеющих заострения и находящихся на „достаточно большом“ расстоянии друг от друга.

В работе [8] для детектирования единичных молекул использовалась спектроскопия, усиленная острием, когда золотое острие приближалось к плоской золотой поверхности на расстояние, примерно равное разме-

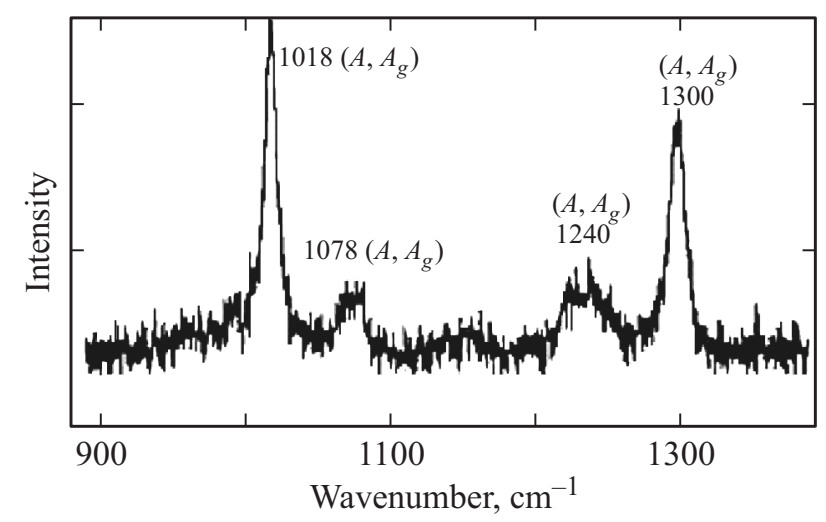

Рис. 5. Спектр ГКР единичных молекул 4,4'-бипиридина, адсорбированных на двумерной решетке из серебряных наночастиц [12].

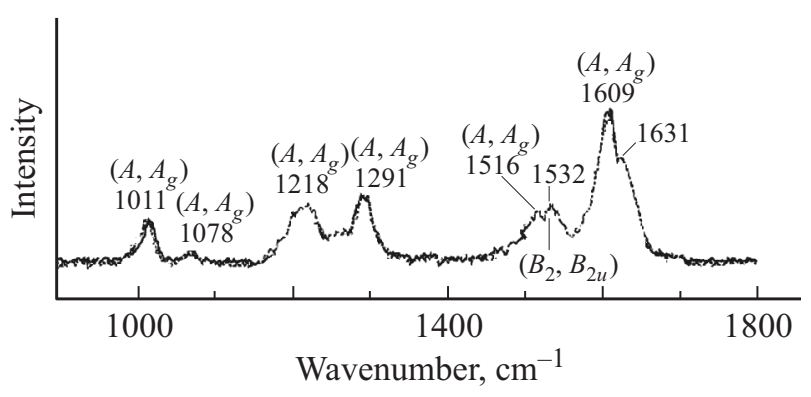

Рис. 6. Спектр единичной молекулы 4,4'-бипиридина, находящейся между золотым острием и плоской поверхностью из золота в геометрии эксперимента TERS [8]. 
ру молекулы. При этом молекула оказывалась в поле своеобразного конденсатора. Регистрация спектров проводилась в более широком интервале от 900 до $1600 \mathrm{~cm}^{-1}$ (рис. 6). Хотя при этом наиболее усиленные линии наблюдались при значениях 1011, 1078, 1218, 1291, 1516 и $1609 \mathrm{~cm}^{-1}$, обусловленных вкладами с единичными неприводимыми представлениями $A_{1}$ или $A_{g}$, в спектре видна очень слабая линия $1532 \mathrm{~cm}^{-1}$, которая может быть отнесена к неприводимым представлениям $B_{2}$ или $B_{2 u}$. Этот факт указывает на то, что хотя основную роль в усилении в данном случае также играет сильное квадрупольное взаимодействие, сильное дипольное взаимодействие в сравнении с последним тоже достаточно велико, что и обусловливает появление в спектре вышеуказанной линии.

\section{4. Заключение}

Таким образом, исследование спектров ГКР 4,4'-бипиридина в режиме определения малого количества или единичных молекул на достаточно удаленных друг от друга наночастицах указывает, что практически все линии, наблюдающиеся в спектрах, обусловлены вкладами, связанными с сильным квадрупольным взаимодействием света с молекулами и соответственно с полно симметричными колебаниями, преобразующимися по единичным неприводимым представлениям $A_{1}$ или $A_{g}$ соответствующих групп симметрии данной молекулы $D_{2}$ или $D_{2 h}$. При этом линии, связанные с квадруполь-дипольным рассеянием типа $\left(Q_{\text {main }}-d_{\alpha}\right)$, и соответственно с колебаниями, преобразующимися по неприводимым представлениям $B_{1}$ или $B_{1 u}, B_{2}$ или $B_{2 u}, B_{3}$ или $B_{3 u}$, описывающим трансформационные свойства остальных дипольных моментов, отсутствуют. Данный факт доказывает сравнительную малую роль сильного дипольного взаимодействия в данных экспериментальных условиях. В случае использования техники TERS, хотя сильное квадрупольное взаимодействие и преобладает над сильным дипольным взаимодействием, последнее, по-видимому, может проявляться в спектрах в виде запрещенных линий, связанных с колебаниями, преобразующимися по неприводимым представлениям, описывающим трансформационные свойства дипольных моментов. Этот результат связан с существенным отличием геометрии эксперимента в сравнении с использованием удаленных друг от друга наночастиц.

\section{Список литературы}

[1] Nie S., Emory S.R. //Science. 1997. V. 275. N 5303. P. 1102.

[2] Kneipp K., Wang Y., Kneipp H., Perelman L.T., Itzkan I., Dasari R.R., Feld M.S. // Phys. Rev. Lett. 1997. V. 78. N 9. P. 1667.

[3] Polubotko A.M. Dipole-Quadrupole Theory of Surface Enhanced Raman Scattering. Nova Science Publishers Inc., 2009. $136 \mathrm{p}$.
[4] Полуботко А.М., Челибанов В.П. // Опт. и спектр. 2015. T. 119. № 4. C. 643. Polubotko A.M., Chelibanov V.P. // Opt. Spectrosc. 2015. V. 119. N 4. P. 664.

[5] Polubotko A.M. // J. Opt. A. 1999. V. 1. P. L18.

[6] Полуботко А.М., Челибанов В.П. // Опт. и спектр. 2016. T. 120. № 1. C. 99. Polubotko A.M., Chelibanov V.P. // Opt. Spectrosc. 2016. V. 120. N 1. P. 86.

[7] Полуботко А.М. // Опт.и спектр. 2013. Т. 114. № 5. С. 46. Polubotko A.M. // Opt. Spectrosc. 2013. V. 114. N 5. P. 696.

[8] Liu Z., Ding S.-Y., Chen Z.-B., Wang X., Tian J.-H., Anema J.R., Zhou X.-S., Wu D.-Y., Mao B.-W., Xu X., Renand B., Tian Z.-Q. // Nature commun. 2011. V. 2. P. 1.

[9] Golovin A.V., Polubotko A.M. // Chem. Phys. Lett. 2016. V. 662. P. 208.

[10] Zhuang Z., Cheng J., Wang X., Zhao B., Han X., Luo Y. // Spectrochim. Acta A. 2007. V. 67. P. 509.

[11] Sawai Y., Takimoto B., Nabika H., Ajito K., Murakoshi K. // J. Am. Chem. Soc. 2007. V. 129. P. 1658.

[12] Takase M., Sawai Y., Nabika H., Murakoshi K. // J. Photochem. Photobiol. A. 2011. V. 221. P. 169 